\title{
The Synchrotron Spectrum of Fast Cooling Electrons Revisited
}

\author{
Jonathan Granot*, Tsvi Piran*, and Re'em Sari ${ }^{\dagger}$ \\ * Racah Institute, Hebrew University, Jerusalem 91904, Israel \\ $\dagger$ Theoretical Astrophysics, California Institute of Technology, Pasadena, CA 91125
}

\begin{abstract}
We discuss the spectrum arising from synchrotron emission by fast cooling electrons when fresh electrons are continually accelerated by a strong blast wave into a power law distribution of energies. The fast cooling spectrum was so far described by four power law segments: $F_{\nu} \propto \nu^{2}, \nu^{1 / 3}, \nu^{-1 / 2}$ and $\nu^{-p / 2}$, divided by three break frequencies: $\nu_{s a}<\nu_{c}<\nu_{m}$. This is valid for a homogeneous electron distribution. However, hot electrons are located right after the shock, while most electrons are farther down stream and have cooled. We show that this spatial distribution introduces a new break frequency, $\nu_{a c}$, in the optically thick regime: $\nu_{a c}<\nu_{s a}<\nu_{c}<\nu_{m}$, and a new spectral slope: $F_{\nu} \propto \nu^{11 / 8}$ for $\nu_{a c}<\nu<\nu_{s a}$, while the familiar $F_{\nu} \propto \nu^{2}$ is obtained only for $\nu<\nu_{a c}$. While the above ordering of the break frequencies is relevant for afterglows with typical parameters in an ISM environment, other possibilities may also be relevant for internal shocks or afterglows in dense circumstellar winds. We discuss possible applications for gamma-ray bursts (GRBs) and their afterglows, in the context of the internal-external shock model. This may explain spectral slopes steeper than $1 / 3$ seen in the $1-10 \mathrm{keV}$ range in some bursts, if $\nu_{s a}$ reaches the X-ray.
\end{abstract}

\section{INTRODUCTION}

The spectrum of GRBs and their afterglows is well described by synchrotron and inverse Compton emission. It is better studied during the afterglow stage, where we have broad band observations. The observed spectrum is in a good agreement with the theory. Within the fireball model, both the GRB and its afterglow are caused by the slowing down of a relativistic flow through shock waves. According to the internal-external shock scenario, the GRB itself arises from internal shocks within the flow, while the afterglow is due to the external shock produced when the flow is decelerated as it sweeps the ambient medium. In this paper we consider fast cooling, where the electrons cool due to radiation losses on a time scale much shorter than the dynamical time of the system.

We consider the synchrotron spectrum of relativistic electrons which are continually accelerated by a strong blast wave into a power law energy distribution: $N\left(\gamma_{e}\right) \propto \gamma_{e}^{-p}$ for $\gamma_{e} \geq \gamma_{m}$. After being accelerated by the shock, the electrons cool down due to radiation losses. On the dynamical time of the system, all electrons cool to a Lorentz factor of $\gamma_{c}$ [1]. The typical synchrotron frequency of an electron

CP526, Gamma-Ray Bursts: 5 $^{\text {th }}$ Huntsville Symposium, edited by R. M. Kippen, et al. (C) 2000 American Institute of Physics 1-56396-947-5/00/\$17.00 
with a randomly oriented velocity is $\nu_{s y n}\left(\gamma_{e}\right)=3 \gamma q_{e} B \gamma_{e}^{2} / 16 m_{e} c$, where $\gamma_{e}, m_{e}$ and $q_{e}$ are its Lorentz factor, mass and electric charge, respectively, $B$ is the magnetic field and $\gamma$ is the bulk Lorentz factor of the flow. The two critical Lorentz factors, $\gamma_{m}$ and $\gamma_{c}$, thereby define two critical frequencies: $\nu_{m}$ and $\nu_{c}$, respectively. In the fast cooling regime, $\gamma_{c} \ll \gamma_{m}$ and therefore $\nu_{c} \ll \nu_{m}$. Another critical frequency is $\nu_{s a}$, below which the system becomes optically thick to self absorption.

The spectrum of a homogeneous distribution of fast cooling electrons had been investigated for $\nu_{s a}<\nu_{c}<\nu_{m}$ [1,2]. It consists of four power law segments: $F_{\nu} \propto \nu^{2}, \nu^{1 / 3}, \nu^{-1 / 2}$ and $\nu^{-p / 2}$, divided by these three critical frequencies. The spectral slope above $\nu_{m}$ is related to the electron injection distribution; the number of electrons with Lorentz factors of the order $\gamma_{e}$ is $\propto \gamma_{e}^{1-p}$ and their energy $\propto \gamma_{e}^{2-p}$. As these electrons cool, they deposit most of their energy into a frequency range of the order of $\nu_{s y n}\left(\gamma_{e}\right) \propto \gamma_{e}^{2}$ and thercforc $F_{\nu} \propto \gamma_{e}^{-p} \propto \nu^{-p / 2}$. In the frequency range $\nu_{c}<\nu<\nu_{m}$ we have contribution from all the electrons in the system as they all cool on the dynamical time. Since the electron's energy is $\propto \gamma_{e}$ and its typical frequency $\propto \gamma_{e}^{2}$, the flux per unit frequency is $\propto \gamma_{e}^{-1} \propto \nu^{-1 / 2}$. At $\nu_{s a}<\nu<\nu_{c}$ we see the synchrotron low frequency tail of the cooled electrons, which is given by $\nu^{1 / 3}$. At $\nu<\nu_{s a}$ the spectrum is self absorbed, and we see the Rayleigh-Jeans portion of the black body spectrum: $\nu^{2}$. According to the Rayleigh-Jeans law, $F_{\nu} \propto \nu^{2} \gamma_{c, t y p}(\nu)$, where $\gamma_{e, t y p}(\nu)$ is the typical thermal Lorentz factor of the electrons emitting at the observed frequency $\nu$. The assumption leading to the $\nu^{2}$ spectral slope below $\nu_{s a}$ is that the relevant temperature is that of the cooled electrons, $\gamma_{e, t y p}(\nu)=\gamma_{c} \propto \nu^{0}$, as these are the electrons which radiate the low energy tail.

We show here that for fast cooling, the self absorbed flux deviates from the $\nu^{2}$ power law due to spatial effects which have been ignored in previous works. We derive the fast cooling spectrum taking into account the inhomogeneous distribution of the electron effective temperatures behind the shock. We provide expressions for the break frequencies and maximal flux density, and discuss the relevance of the new spectral slope, $F_{\nu} \propto \nu^{11 / 8}$, both to the prompt emission and the afterglow.

\section{FAST COOLING SPECTRUM}

The shape of the fast cooling spectrum is determined by the relative ordering of the break frequencies: $\nu_{s a}, \nu_{c}$ and $\nu_{m}$. For fast cooling, $\nu_{c} \ll \nu_{m}$, leaving three possibilities for this ordering, each of which gives rise to a different spectrum.

We begin the "canonical" situation for which the homogeneous spectrum was described above: $\nu_{s a}<\nu_{c}<\nu_{m}$. We denote this by case (1). The optically thin $\left(\nu>\nu_{s a}\right)$ part of the spectrum is the same for the homogeneous and inhomogeneous electron distributions behind the shock, since all the emitted radiation escapes the system, and the location of the emitting electrons becomes irrelevant. Immediately behind the shock there is a thin layer where most electrons have not yet cooled significantly, followed by a much wider layer where all electrons at a given distance, $l^{\prime}$, behind the shock (in the local frame) have cooled down to $\gamma_{e} \propto 1 / l^{\prime}$. In the optically thick regime, most of the photons which escape the system are emitted at 
an optical depth close to unity: $\tau_{\nu} \sim 1$. Therefore, $\gamma_{e, t y p}(\nu)$ must be evaluated at the place where $\tau_{\nu}=1$. An optical depth of unity is obtained at a distance $l^{\prime}$ behind the shock where the optically thin emission equals the optically thick emission:

$$
n^{\prime} l^{\prime} \gamma^{2}\left(P_{\nu, \max } / 4 \pi\right)\left[\nu / \nu_{s y n}\left(\gamma_{e}\right)\right]^{1 / 3}=\left(2 \nu^{2} / c^{2}\right) \gamma \gamma_{e} m_{e} c^{2}
$$

where $n^{\prime}$ is the proper number density and $P_{\nu, \max } \propto \gamma_{e}^{0}$ is the peak spectral power of an clectron. Since $\nu_{s y n}\left(\gamma_{e}\right) \propto \gamma_{e}^{2}$, and within the wide layer of cooled electrons, $l^{\prime} \propto 1 / \gamma_{e}$, equation (1) implies that $\gamma_{e, t y p}(\nu)=\gamma_{e} \propto \nu^{-5 / 8}$, and according to the Rayleigh-Jeans law, $F_{\nu} \propto \nu^{11 / 8}$.

The new spectral slope, $F_{\nu} \propto \nu^{11 / 8}$, begins at $\nu_{s a}$, as the system becomes optically thick, and extends to lower frequencies. However, at sufficiently low frequencies $\left(\nu<\nu_{a c}\right), \tau_{\nu}=1$ is reached within the thin layer just behind the shock where the electrons have not cooled significantly, implying $\gamma_{e, t y p}(\nu)=\gamma_{m} \propto \nu^{0}$ and $F_{\nu} \propto \nu^{2}$. The spectrum in case (1) is shown in the upper frame of Figure 1, and the break frequencies and maximal flux density are given by

$$
\begin{aligned}
\nu_{a c} & =\left(2^{7} m_{e} 4 c^{13} \gamma^{5} n^{3} / 3^{7} \pi^{3} B^{4} q_{e}^{4} \gamma_{m}^{8}\right)^{1 / 5}=1.3 \times 10^{8} \mathrm{~Hz} n_{2}^{3 / 5} \gamma_{40} B_{10}^{-4 / 5} \gamma_{m, 4}^{-8 / 5}, \\
\nu_{s a}^{(1)} & =\left(\sigma_{T}^{8} B^{12} c^{5} t_{d y n}^{8} \gamma^{13} n^{\prime 3} / 3^{15} 2 \pi^{11} m_{e}^{4} q_{e}^{4}\right)^{1 / 5}=7.3 \times 10^{10} \mathrm{~Hz}_{2}^{3 / 5} \gamma_{40}^{13 / 5} B_{10}^{12 / 5} t_{d y n, 3}^{8 / 5}, \\
\nu_{c} & =27 \pi^{2} m_{e} c q_{e} / 4 \sigma_{T}^{2} \gamma B^{3} t_{d y n}^{2}=4.9 \times 10^{13} \mathrm{~Hz} \gamma_{40}^{-1} B_{10}^{-3} t_{d y n, 3}^{-2}, \\
\nu_{m} & =3 q_{e} B \gamma \gamma_{m}^{2} / 16 m_{e} c=1.3 \times 10^{17} \mathrm{~Hz} \gamma_{40} B_{10} \gamma_{m, 4}^{2}, \\
F_{\nu, \text { max }}^{(1)} & =2 \sigma_{T} m_{e} c^{2} N_{e} \gamma B / 9 \pi^{2} q_{e} D^{2}=100 \mathrm{mJy} N_{e, 52} D_{28}^{-2} \gamma_{40} B_{10},
\end{aligned}
$$

where $n_{2}=n^{\prime} / 100 \mathrm{~cm}^{-3}, \gamma_{40}=\gamma / 40, B_{10}=B / 10 \mathrm{G}, \gamma_{m, 4}=\gamma_{m} / 10^{4}, t_{d y n, 4}=$ $t_{d y n} / 10^{4} \mathrm{sec}, D=D_{28} 10^{28} \mathrm{~cm}$ is the distance to the source, and $N_{e}=N_{e, 52} 10^{52}$ is the number of radiating electrons, assuming an isotropic flow. This parameterization reflects typical values during the early afterglow. The supcrscript ${ }^{(i)}$ labels the specific case under consideration.

We now turn to case (2), where $\nu_{c}<\nu_{s a}<\nu_{m}$. Now, $\nu_{c}$ is unimportant as it is within the optically thick regime, and the spectrum is given in the middle frame of Figure 1. The maximal flux density $F_{\nu, \max }^{(2)}$ is now reached at $\nu_{s a}^{(2)}$, and both quantities are given by

$$
\nu_{s a}^{(2)}=\left(\nu_{s a}^{(1)}\right)^{5 / 9} \nu_{c}^{4 / 9} \quad, \quad F_{\nu, \max }^{(2)}=F_{\nu, \max }^{(1)}\left(\nu_{c} / \nu_{s a}^{(1)}\right)^{5 / 18} .
$$

The last case (3) occurs when $\nu_{c}<\nu_{m}<\nu_{s a}$. Now the system becomes optically thick due to absorption from a very thin layer behind the shock front, well within the thin layer where most electrons have not cooled significantly. Therefore, both $\nu_{a c}$ and $\nu_{c}$ are now irrelevant, since the inner parts where these frequencies are important are not visible. Furthermore, the initial electron distribution may be used to evaluate $\gamma_{e, t y p}(\nu)$. Thus, $\gamma_{e, t y p}(\nu) \propto \gamma_{m} \propto \nu^{0}$ at $\nu<\nu_{m}$, implying $F_{\nu} \propto \nu^{2}$, while at $\nu_{m}<\nu<\nu_{s a}$ the emission is dominated by electrons with $\nu_{s y n}\left(\gamma_{e}\right) \sim \nu$, implying $\gamma_{e, t y p}(\nu) \propto \nu^{1 / 2}$ and $F_{\nu} \propto \nu^{5 / 2}$. The resulting spectrum is shown in the lower frame of Figure 1, where $\nu_{m}$ remains unchanged while 


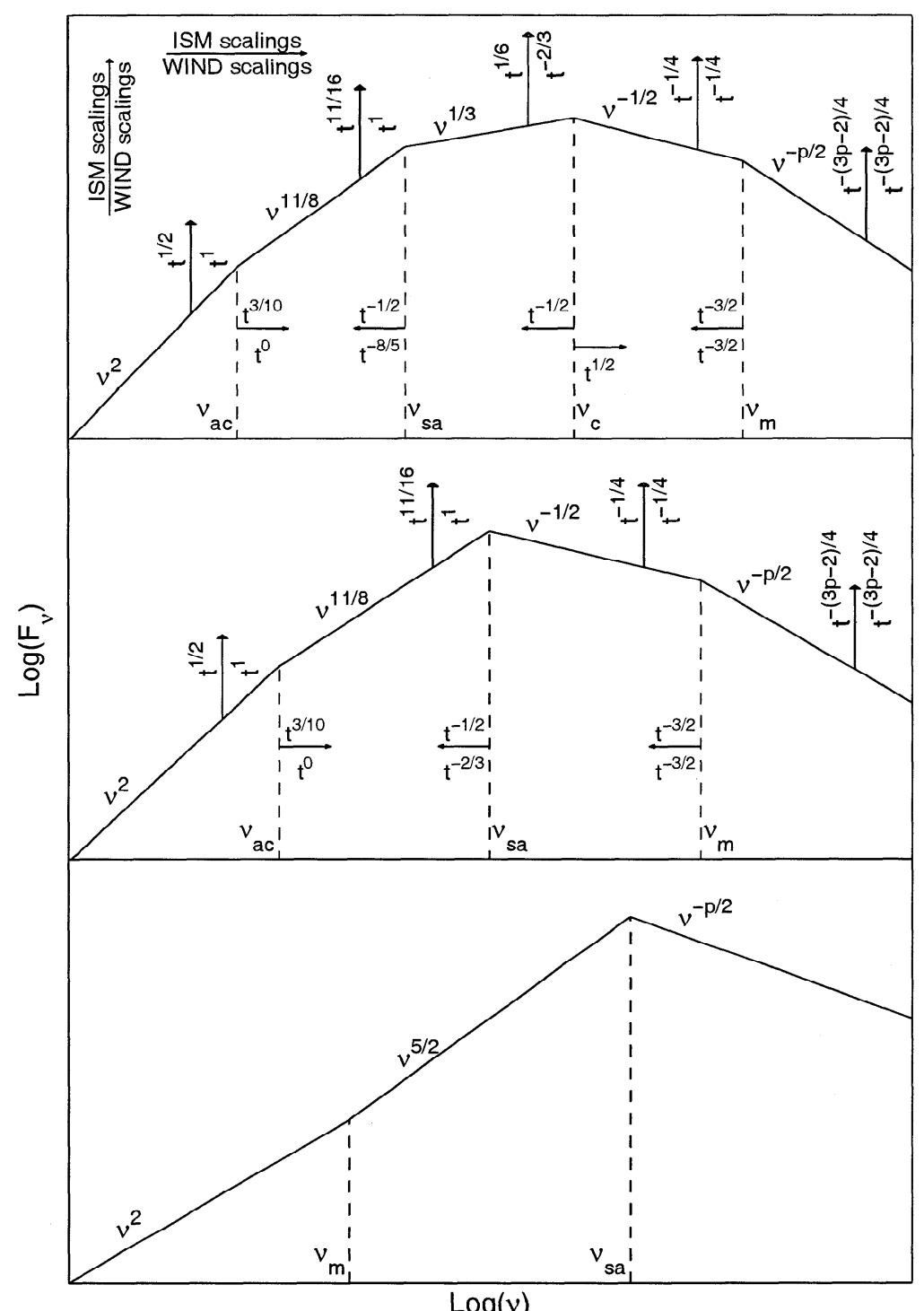

FIGURE 1. Fast cooling synchrotron spectra from a shock injected power law electron distribution. The shape of the spectrum is determined by the ordering of the self absorption frequency, $\nu_{s a}$, with respect to $\nu_{c}<\nu_{m}$. There are three possible shapes for the spectrum, corresponding to $\nu_{s a}<\nu_{c}, \nu_{c}<\nu_{s a}<\nu_{m}$ and $\nu_{s a}>\nu_{m}$, from top to bottom. The temporal scalings for an external shock, both for an ISM and stellar wind (denoted WIND) environments, are given only in the first two frames, since the last (bottom) frame is unlikely. 


$$
\nu_{s a}^{(3)}=\left[\left(\nu_{s a}^{(1)}\right)^{10 / 3} \nu_{c}^{8 / 3} \nu_{m}^{p-1}\right]^{\frac{1}{(p+5)}} \quad, \quad F_{\nu, \max }^{(3)}=F_{\nu, \max }^{(1)}\left[\left(\nu_{s a}^{(1)}\right)^{-p / 3} \nu_{c}^{\frac{(3-p)}{6}} \nu_{m}^{\frac{(p-1)}{2}}\right]^{\frac{5}{(p+5)}}
$$

\section{APPLICATION TO GRBS}

For afterglows in an ISM surrounding, fast cooling typically last for one hour, and the spectrum is of the first type (upper frame in Figure 1). If the surrounding is a stellar wind of a massive progenitor, the fast cooling stage typically lasts for about a day [3], during which the spectrum is of the second kind (middle frame in Figure 1) for the first $1-2$ hours, and then switches to the spectrum of the first kind. The time dependence of the break frequencies and flux density for an afterglow in an ISM or wind environment is given in Figure 1.

During the prompt gamma-ray emission, both the highly variable temporal structure of most bursts and the requirement of a reasonable radiative efficiency $[4,5]$ suggest fast cooling. All three cases for the spectrum are possible. The spectra of the third type (lower frame of Figure 1) is likely to occur with slow cooling conditions $\left(\nu_{m}<\nu_{c}\right)$, but this should not effect the spectrum as long as $\nu_{c}<\nu_{s a}$.

\section{DISCUSSION}

The synchrotron spectrum of fast cooling electrons is summarized in Figure 1. We find three possible spectra, depending on the ordering of $\nu_{s a}$ with respect to $\nu_{c}<\nu_{m}$. Two of these cases include a new self absorption regime where $F_{\nu} \propto \nu^{11 / 8}$.

During the initial fast cooling stage of the afterglow, the system is typically optically thick in the radio and optically thin in the optical and X-ray. We therefore expect the new feature, $F_{\nu} \propto \nu^{11 / 8}$, to be observable only in the radio band during the afterglow. This requires fast radio observations, $\lesssim 1$ hour (day) for an ISM (stellar wind) environment, which may become possible after the launch of HETE-2.

During internal shocks, fast cooling conditions are expected, and a spectrum of the first or second type (upper or middle frames of Figure 1) is most likely. If the self absorption frequency reaches the $\mathrm{X}$-ray, this may explain the observed spectral slopes greater than $1 / 3$ (photon number slope larger than $-2 / 3$ ), in the $1-10 \mathrm{keV}$ range [6-8] seen in some bursts.

\section{REFERENCES}

1. Sari, R., Piran, T. \& Narayan, R., ApJ 497, L17 (1998).

2. Sari, R. \& Piran, T., ApJ 520, 641 (1999).

3. Chevalier, R.A., \& Li, Z.Y., astro-ph9908272 (1999).

4. Sari, R. \& Piran, T., ApJ 485, 270 (1997).

5. Fenimore, E. et al., $A p J$ 473, 998 (1996).

6. Preece, R.D. et al., Ap.J 496, 849 (1998).

7. Crider, A. et al., $A p J$ 479, L39 (1998).

8. Strohmayer, T.E. et al., ApJ 500, 873 (1998). 